KCL-MTH-04-08

ITP-Budapest Report No. 611

hep-th/0406139

\title{
A nonperturbative study of phase transitions in the multi-frequency sine-Gordon model
}

\author{
Gábor Zsolt Tóth \\ 8th July 2018 \\ Mathematics Department, \\ King's College London, Strand, London WC2R 2LS, U.K. ${ }^{1}$ \\ HAS Theoretical Physics Research Group, \\ Institute for Theoretical Physics, Eötvös University, \\ H-1117 Budapest, Pázmány Péter Sétány 1/A, Hungary \\ tgzs@ludens .elte.hu
}

\begin{abstract}
The phase spaces of the two- and three-frequency sine-Gordon models are examined in the framework of truncated conformal space approach. The focus is mainly on a tricritical point in the phase space of the three-frequency model. We give substantial evidence that this point exists. We also find the critical line in the phase space and present TCSA data showing the change of the spectrum on the critical line as the tricritical endpoint is approached. We find a few points of the line of first order transition as well.
\end{abstract}

PACS: 64.60.Fr; 11.10.Kk

Keywords: non-integrable quantum field theory, sine-Gordon model, phase transition, Ising model, tricritical Ising model, truncated conformal space approach, finite size effects

\section{Introduction}

The sine-Gordon model in $(1+1)$ dimensions has attracted interest long time ago for the reason that it appears in several areas of physics, nevertheless it is an integrable field theory, that can be used to study non-perturbative quantum field theory phenomena. The areas of application include statistical mechanics of one-dimensional quantum spin chains

\footnotetext{
${ }^{1}$ until 31th October 2004
} 
and nonlinear optics among many others - see the introduction of [1] for a representative list with references.

In the present paper we investigate a non-integrable extension of the sine-Gordon model called multi-frequency sine-Gordon model, in which the scalar potential consists of several cosine terms with different frequencies. It is suggested in [1] that this model can be used to give more refined approximation to some of the physical situations where the ordinary sine-Gordon model can be used. A feature of the multi-frequency model that is new compared to the usual sine-Gordon model is - apart form non-integrability - that phase transition can occur as the coupling constants are tuned. We concentrate our attention to this property. It should be noted that such a phase transition is related to the evolution of the particle spectrum of the theory as the coupling constants vary, and we shall use the massgap and other characteristics of the energy spectrum as order parameter.

Our investigation is a continuation of the work done on the double-frequency case in [3. We use the truncated conformal space approach (TCSA), which is a non-perturbative numerical method to compute the lowest levels of the spectrum of the Hamiltonian operator of perturbed conformal field theories in finite volume. The applicability and reliability of this method was thoroughly investigated in [3], and it was shown that the existence, nature and location of the phase transition can be established by this method, although rather large number of dimensions are needed for satisfactory precision. In particular, the existence and location of an Ising type transition was established in the double-frequency model (DSG) for the ratio $1 / 2$ of the frequencies, verifying a prediction by [1] based on perturbation theory and classical arguments. We extend these investigations to the ratio $1 / 3$ and to the three-frequency model (at the ratio $1 / 2 / 3$ of the frequencies), in which a tricritical point and first order transition are expected to be found. The numerical nature of the TCSA makes it necessary to choose specific values for the frequencies.

After introducing the multi-frequency sine-Gordon model and describing basic properties of it in Section 2, we briefly review the TCSA framework for the model (which is the framework described in [3] adapted to the multi-frequency model) in Section 3. In Section 4 we give a description of the phase structure of the classical two- and three-frequency model, which serves as a reference for the investigations in quantum theory. The $n$ frequency case is also considered briefly. Exact and elementary analytic methods can be applied to the classical case, and the results are more general than in the quantum case. Section 5 is devoted to theoretical considerations on the signatures of 1 st and 2 nd order phase transitions in the framework of perturbed conformal field theory in finite volume. Most of these considerations, which are necessary for the evaluation of the TCSA data, can also be found in [3]. Finally, in Section [6] and 7 we present the results we obtained by TCSA on the phase structure of the two- and three-frequency model, which are the main results in the paper. A summary of the results and conclusions are given in Section 8 


\section{The multi-frequency sine-Gordon model}

\subsection{The definition and basic properties of the model}

The action of the multi-frequency sine-Gordon model (MSG) is

$$
\mathcal{A}_{M S G}=\int \mathrm{d} t \int \mathrm{d} x\left(\frac{1}{2} \partial_{\mu} \Phi \partial^{\mu} \Phi-V(\Phi)\right)
$$

where

$$
V(\Phi)=\sum_{i}^{n} \mu_{i} \cos \left(\beta_{i} \Phi+\delta_{i}\right)
$$

is the potential, which contains $n$ cosine terms. $\Phi$ is a real scalar field defined on the two-dimensional Minkowski space $\mathbb{R}^{1+1}, \beta_{i} \in \mathbb{R}$ are the frequencies, $\beta_{i} \neq \beta_{j}$ if $i \neq j, \mu_{i}$ are the coupling constants (of dimension mass ${ }^{2}$ at the classical level) and $\delta_{i} \in \mathbb{R}$ are the phases in the terms of the potential.

Two cases can be distinguished according to the periodicity properties of the potential. The first one is the rational case, when $V(\Phi)$ is a trigonometric polynomial: the ratios of the frequencies $\beta_{i}$ are rational and the potential is periodic. Let the period of the potential be $2 \pi r$ in this case. The target space of the field $\Phi$ can be compactified:

$$
\Phi \equiv \Phi+2 k r \pi
$$

where $k \in \mathbb{N}$ can be chosen arbitrarily. The model obtained in this way is called the $k$-folded MSG. The well-known classical sine-Gordon model corresponds to $n=1, k=1$.

The other case is the irrational one, when the potential is not periodic and no such folding can be made. The irrational case is much more complicated than the rational one, so we restrict our attention to the rational case in the present paper. We remark here only that although $V(\Phi)$ always has a finite infimum, it does not necessarily admit an absolute minimum. The potential $V(\Phi)$ can always be written uniquely as a sum $V(\Phi)=V_{1}(\Phi)+V_{2}(\Phi)+\ldots+V_{k}(\Phi)$, where the terms $V_{1}, \ldots, V_{k}$ are periodic but any sum of any of these terms is not periodic. $V(\Phi)$ has an absolute minimum if and only if $V_{1}(\Phi), \ldots, V_{k}(\Phi)$ have a common absolute minimum. This occurs for special choice of the $\delta_{i}$, if the values of $\beta_{i}$ are given. In particular, if $\beta_{i} / \beta_{j}$ are irrational for all $i \neq j$ and $\mu_{i}<0$ for all $i$, then $V(\Phi)$ has a absolute minimum if and only if $\frac{\delta_{i}}{\beta_{i}}-\frac{\delta_{j}}{\beta_{j}}=\frac{2 \pi b_{i}}{\beta_{i}}-\frac{2 \pi b_{j}}{\beta_{j}}$ is satisfied with some numbers $b_{i} \in \mathbb{Z}$, which is equivalent to the case $\delta_{i}=0$ for all $i$. See and [1] and [3] for further remarks on the irrational case.

At the quantum level the theory can be regarded as a perturbed conformal field theory:

$$
\mathcal{A}_{M S G}=\mathcal{A}_{C F T}+\mathcal{A}_{\text {pert }},
$$

where

$$
\mathcal{A}_{C F T}=\int \mathrm{d} t \int \mathrm{d} x \frac{1}{2} \partial_{\mu} \Phi \partial^{\mu} \Phi
$$


which is the action of the free scalar particle of zero mass, and

$$
\mathcal{A}_{\text {pert }}=\int \mathrm{d} t \int \mathrm{d} x(-V(\Phi))=-\frac{1}{2} \int \mathrm{d} t \int \mathrm{d} x \sum_{i=1}^{n}\left(\mu_{i} e^{i \delta_{i}} V_{\beta_{i}}+\mu_{i} e^{-i \delta_{i}} V_{-\beta_{i}}\right),
$$

where $V_{\omega}$ denotes the vertex operator

$$
V_{\omega}=: e^{i \omega \Phi}:
$$

which is a primary field with conformal dimensions

$$
\Delta_{\omega}^{ \pm}=\Delta_{\omega}=\frac{\omega^{2}}{8 \pi}
$$

in the unperturbed (conformal) field theory. The upper index \pm corresponds to the left/right conformal algebra and : : denotes the conformal normal ordering. The dimensions of the couplings at the quantum level are

$$
\left[\mu_{i}\right]=(\text { mass })^{2-2 \Delta_{i}}, \quad \Delta_{i} \equiv \Delta_{\beta_{i}} .
$$

The perturbing operators are relevant only if

$$
\beta_{i}^{2}<8 \pi,
$$

we restrict ourselves to this case. We also assume that

$$
\beta_{i}^{2}<4 \pi
$$

which is a necessary and sufficient condition for the model to be free from ultraviolet divergencies in the perturbed conformal field theory framework [8, 1, 3].

The model has a massgap in general, and it is clear that phase transitions occur in the classical version of the model as the coupling constants are tuned (assuming that $n>1$ ). It is also expected that there are topologically charged solutions/states in the model [1]. In the present paper we shall investigate the sector with zero topological charge, which is sufficient for our purposes. We also restrict ourselves to 1 -folded models $(k=1)$, as it is natural to expect that in infinite volume a folding number $k \neq 1$ results simply in a $k$-fold multiplication of the spectrum corresponding to $k=1$.

\section{The Truncated Conformal Space Approach}

The matrix elements of the total Hamiltonian operator $H=H_{C F T}+H_{\text {pert }}$ can be calculated explicitly (and exactly) between any two elements of the Hilbert space $\mathcal{H}_{C F T}$ of the conformal field theory. Restricting to a finite dimensional subspace $\mathcal{H}_{0}$ of $\mathcal{H}_{C F T}$ by 
introducing an upper conformal energy cutoff $H$ becomes a finite matrix, which can be diagonalized numerically to get an approximation of the spectrum. As this description shows, TCSA is a variational method.

The $k$-folded free boson as a conformal field theory contains the following primary fields:

$$
V_{p, \bar{p}}(z, \bar{z})=: \exp \left[i p \phi_{C F T}(z)+i \bar{p} \bar{\phi}_{C F T}(\bar{z})\right]:
$$

with conformal dimensions $\Delta^{+}=\frac{p^{2}}{8 \pi}, \Delta^{-}=\frac{\bar{p}^{2}}{8 \pi}$, where $p=\frac{n}{r}+2 \pi r m, \bar{p}=\frac{n}{r}-2 \pi r m$, $n, m \in \mathbb{Z}$,

$$
\Phi_{C F T}(x, t)=\phi_{C F T}(x-t)+\bar{\phi}_{C F T}(x+t) .
$$

$\mathcal{H}_{C F T}$ is spanned by the states $|p, \bar{p}\rangle=\lim _{z, \bar{z} \rightarrow 0} V_{p, \bar{p}}(z, \bar{z})|0\rangle(|0,0\rangle \equiv|0\rangle)$ and $a_{n_{1} \ldots} \bar{a}_{m_{1} \ldots}|p, \bar{p}\rangle$, where $a_{n_{i}}$ and $\bar{a}_{m_{i}}$ are creating operators of Fourier modes on the "conformal plane". The conformal generators $L_{0}$ and $\bar{L}_{0}$ are diagonal in this basis. The basis of $\mathcal{H}_{0}$ is obtained by taking those elements $|v\rangle$ of the basis above which satisfy the truncation condition

$$
\frac{\left\langle v\left|\frac{L}{2 \pi} H_{C F T}\right| v\right\rangle}{\langle v \| v\rangle}<e_{c u t} .
$$

$e_{c u t}$ is the dimensionless upper conformal energy cutoff and $L$ is the volume of space. We restrict ourselves to the sector with zero topological charge $(p=\bar{p})$, this being the sector containing the ground state(s) and the relevant information for the problem treated in this work. In the sector containing the ground state(s) the Lorentz spin of all the states is also zero, so we also impose the condition $\left(L_{0}-\bar{L}_{0}\right)|v\rangle=0$. (The operator $L_{0}-\bar{L}_{0}$ commutes with $H$ and $H_{C F T}$ as well). We remark that $e_{c u t}$ serves as an ultraviolet cutoff.

The matrix elements of $H$ between two elements $|a\rangle$ and $|b\rangle$ of the basis of $\mathcal{H}_{C F T}$ above are given by

$$
\begin{gathered}
\left(\frac{H}{M}\right)_{a b}=\frac{2 \pi}{l}\left(L_{0}+\bar{L}_{0}-\frac{c}{12}\right)_{a b} \\
+\frac{2 \pi}{l} \sum_{j=1}^{n} \operatorname{sgn}\left(\mu_{j}\right) \kappa_{j}\left(\frac{M_{j}}{M}\right)^{x_{j}} \frac{l^{x_{j}}}{2(2 \pi)^{x_{j}-1}} e^{i \delta_{j}}\left(V_{\beta_{j}, \beta_{j}}(1,1)\right)_{a b} \delta_{\Delta_{a}-\bar{\Delta}_{a}, \Delta_{b}-\bar{\Delta}_{b}} \\
+\frac{2 \pi}{l} \sum_{j=1}^{n} \operatorname{sgn}\left(\mu_{j}\right) \kappa_{j}\left(\frac{M_{j}}{M}\right)^{x_{j}} \frac{l^{x_{j}}}{2(2 \pi)^{x_{j}-1}} e^{-i \delta_{j}}\left(V_{-\beta_{j},-\beta_{j}}(1,1)\right)_{a b} \delta_{\Delta_{a}-\bar{\Delta}_{a}, \Delta_{b}-\bar{\Delta}_{b}},
\end{gathered}
$$

where $M$ is a mass scale of the theory given below, $l=L M$ is the dimensionless volume, $x_{j}=2-2 \Delta_{j}, \Delta_{j} \equiv \Delta_{\beta_{j}} ; \Delta_{a}, \bar{\Delta}_{a}, \Delta_{b}, \bar{\Delta}_{b}$ are the conformal weights of the states $|a\rangle$ and $|b\rangle, c$ is the central charge of the conformal theory ( $c=1$ in the present case), and we have made a replacement corresponding to

$$
\left|\mu_{j}\right|=\kappa_{j} M_{j}^{x_{j}}
$$


The "interpolating" mass scale $M$ is

$$
M=\sum_{j} \eta_{j} M_{j}
$$

where

$$
\eta_{j}=\frac{\left|\mu_{j}\right|^{1 / x_{j}}}{\sum_{i}\left|\mu_{i}\right|^{1 / x_{i}}}
$$

are the dimensionless coupling constants (of which only $n-1$ are independent). (3) implies that $\eta_{j} \in[0,1], \sum_{j} \eta_{j}=1$. $M$ depends smoothly on the $\eta_{j}$-s. The precise expression for $\kappa$ is not essential for our problem, we need only that $\kappa$ depends on $\Delta$ only and that it is dimensionless. Following [3] we used the formula of [7]:

$$
\kappa_{j}=\frac{2 \Gamma\left(\Delta_{j}\right)}{\pi \Gamma\left(1-\Delta_{j}\right)}\left(\frac{\sqrt{\pi} \Gamma\left(\frac{1}{x_{j}}\right)}{2 \Gamma\left(\frac{\Delta_{j}}{x_{j}}\right)}\right)^{x_{j}} .
$$

The formula (2) is written in terms of dimensionless quantities, and the volume $(l)$ dependence of $H / M$ is also explicit. It is clear from (2) that TCSA gives an exact result if $l \rightarrow 0$ (assuming (10) and this limit of the theory is the conformal theory (the massless free boson), and the accuracy of the TCSA spectrum decreases at fixed $e_{\text {cut }}$ as $l \rightarrow \infty$. For very large values of $l$ the $l$-dependence of the spectrum of the TCSA Hamiltonian is power-like, as it is determined directly by the $l^{x_{j}-1}$ coefficients in (2). The TCSA Hamiltonian cannot be considered as good approximation for these values of $l$. Increasing the value of $e_{c u t}$ generally increases accuracy, and the TCSA is expected to give better approximation for the lower lying energy levels than for the higher ones.

We denote the (dimensionless) energy levels of $H / M$ in volume $l$ by $e_{i}(l), i=0,1,2, \ldots$, and $e_{0} \leq e_{1} \leq e_{2} \leq \ldots$ if not stated otherwise. We shall draw conclusions about the spectrum at $l=\infty$ from the behaviour of the functions $e_{i}(l)$ for low values of $i$ and moderately large values of $l$.

We refer to [3, 4, 5] for more details on the TCSA framework.

\section{Phase structure in the classical limit}

\subsection{Phase structure of the two-frequency model in the classical limit}

The Lagrangian density takes the following form in the two-frequency case:

$$
\mathcal{L}=\frac{1}{2} \partial_{\mu} \Phi \partial^{\mu} \Phi-\mu \cos (\beta \Phi)-\lambda \cos (\alpha \Phi+\delta),
$$


where

$$
\frac{\beta}{\alpha}=\frac{n}{m} \neq 1
$$

$n$ and $m$ are coprimes (and the folding number equals to one).

Proposition 1: Assume that $\mu, \lambda \neq 0$. Then the following three cases can be distinguished:

a.) If the function $V(\Phi)$ is symmetric with respect to the reflection $\Phi \mapsto 2 \Phi_{0}-\Phi$, where $\Phi_{0}$ is a suitable constant, and $n, m>1$, then $V$ has two absolute minima, which are mapped into each other by the reflection. We remark that it depends on the value of $\delta$ whether $V$ has this symmetry or not, and it is easy to give a criterion for the existence of this symmetry in terms of $n, m$ and $\delta$.

b.) If $V$ is symmetric with respect to a reflection as in case a., but $n=1$ or $m=1$, then $V$ has one or two absolute minima depending on the values of $\mu$ and $\lambda$. In this case, assuming that $n=1, V$ can be brought to the form

$$
V(\Phi)=-|\mu| \cos (\beta \Phi)+|\lambda| \cos (m \beta \Phi)
$$

by an appropriate shift of $\Phi$. $V$ has two absolute minima if $|\lambda / \mu|>1 / m^{2}$, and one absolute minimum if $|\lambda / \mu| \leq 1 / \mathrm{m}^{2}$. The two absolute minima are mapped into each other by the reflection. The second derivative of $V$ is nonzero at the minima if $|\lambda / \mu| \neq 1 / m^{2}$, but it is zero if $|\lambda / \mu|=1 / \mathrm{m}^{2}$. In the latter case, the fourth derivative of $V$ at the minimum is nonzero. The two minima of $V$ merge and the value of the second derivatives of $V$ at the two minima tends to zero as $|\lambda / \mu|$ approaches $1 / m^{2}$ from above.

c.) If $V$ does not satisfy the requirements of a) and b), then $V$ has a single absolute minimum.

We omit the proof of this proposition, which is elementary, although long and not completely trivial because of the arbitrariness of $n$ and $m$.

If $\mu$ or $\lambda$ equals to zero, then $V$ is periodic and has $m$ or $n$ absolute minima, respectively. See [4] for a detailed investigation of these (integrable) limiting cases.

The phases of the classical model are determined by the behaviour of absolute minima of $V(\Phi)$ as the value of the coupling constants vary. In particular, Proposition 1 implies that the phase structure of the two-frequency model is the following:

The model exhibits an Ising-type second order phase transition at the critical value

$$
\eta_{c}=\frac{m}{1+m}
$$

of the dimensionless coupling constant $\eta=\sqrt{|\mu|} /(\sqrt{|\mu|}+\sqrt{|\lambda|})$ if $n=1$ and $V$ has the $\mathbb{Z}_{2}$-symmetry introduced above. This critical point separates two massive phases with unbroken and spontaneously broken $\mathbb{Z}_{2}$-symmetry. Equivalent statement can be made if $m=1$. If $V$ is not symmetric, then there is only one massive phase with nondegenerate ground state. If $m, n \neq 1$ and $V$ is symmetric, then there is one massive phase with 
doubly degenerate ground state (i.e. the reflection symmetry is spontaneously broken). In the limiting cases $\eta=0$ and $\eta=1$ the model is massive and has spontaneously (and completely) broken $\mathbb{Z}_{n}$ or $\mathbb{Z}_{m}$ symmetry.

\subsection{Phase structure of the three-frequency model in the classical limit}

A complete description of the behaviour of the absolute minima of $V$ for all values of the parameters becomes excessively difficult in the three- and higher-frequency cases, so we restrict our attention to particular values. The potential in the three-frequency case is

$$
V(\Phi)=\mu_{1} \cos \left(\beta_{1} \Phi\right)+\mu_{2} \cos \left(\beta_{2} \Phi+\delta_{2}\right)+\mu_{3} \cos \left(\beta_{3} \Phi+\delta_{3}\right) .
$$

We choose the frequency ratios $3: 2: 1$, i.e.

$$
\beta_{1}=\beta, \quad \beta_{2}=\frac{2}{3} \beta, \quad \beta_{3}=\frac{1}{3} \beta .
$$

This three-frequency model has a tricritical point if and only if $\delta_{2}=\delta_{3}=0$ (and also in a few equivalent cases), in this case $V$ is symmetric with respect to the reflection $\Phi \mapsto-\Phi$. In the tricritical point the absolute minimum of $V$ can be located only at 0 or $\pi$. The two cases are equivalent, we consider the case when the location of the absolute minimum is 0 . The tricritical point in this case is located at

$$
\frac{\mu_{1}}{\mu_{2}}=-\frac{1}{6}, \quad \frac{\mu_{1}}{\mu_{3}}=\frac{1}{15} .
$$

In this point $V^{(6)}(0) \neq 0$. (The upper index ${ }^{(6)}$ denotes the sixth derivative with respect to $\Phi$.) $V^{(6)}(0)>0$ requires $\mu_{1}, \mu_{3}<0$ and $\mu_{2}>0$. We restrict ourselves to this domain and to the values $\delta_{2}=\delta_{3}=0$.

The phase diagram is shown in Figure 1. The points of the diagram correspond to the values of the pair $\left(\eta_{1}, \eta_{2}\right)$ of dimensionless parameters. The allowed values constitute the left lower triangle, the straight line joining $(0,1)$ and $(1,0)$ corresponds to $\eta_{3}=0$. The tricritical point is denoted by $t$, it is located at

$$
\left(\frac{1}{1+\sqrt{6}+\sqrt{15}}, \frac{\sqrt{6}}{1+\sqrt{6}+\sqrt{15}}\right) \approx(0.1365,0.3345) .
$$

At $t V$ has one single and absolute minimum (at $\Phi=0)$. Phase transition occurs when the lines 5 and 3 shown in the phase diagram are crossed. Second order Ising-type phase transition occurs on 5 and first order phase transition occurs on 3 . The domain $A \cup$ $B \cup F$ corresponds to a massive $\mathbb{Z}_{2}$-symmetric phase (with unique ground state). The domain $E \cup C$ corresponds to a massive phase with spontaneously broken $\mathbb{Z}_{2}$-symmetry. Characteristic shapes of the potential in the various domains and on the various lines of the phase diagram can be seen in Figure 2. Data applying to the quantum case are also shown in Figure 1, they will be explained in subsequent sections. 


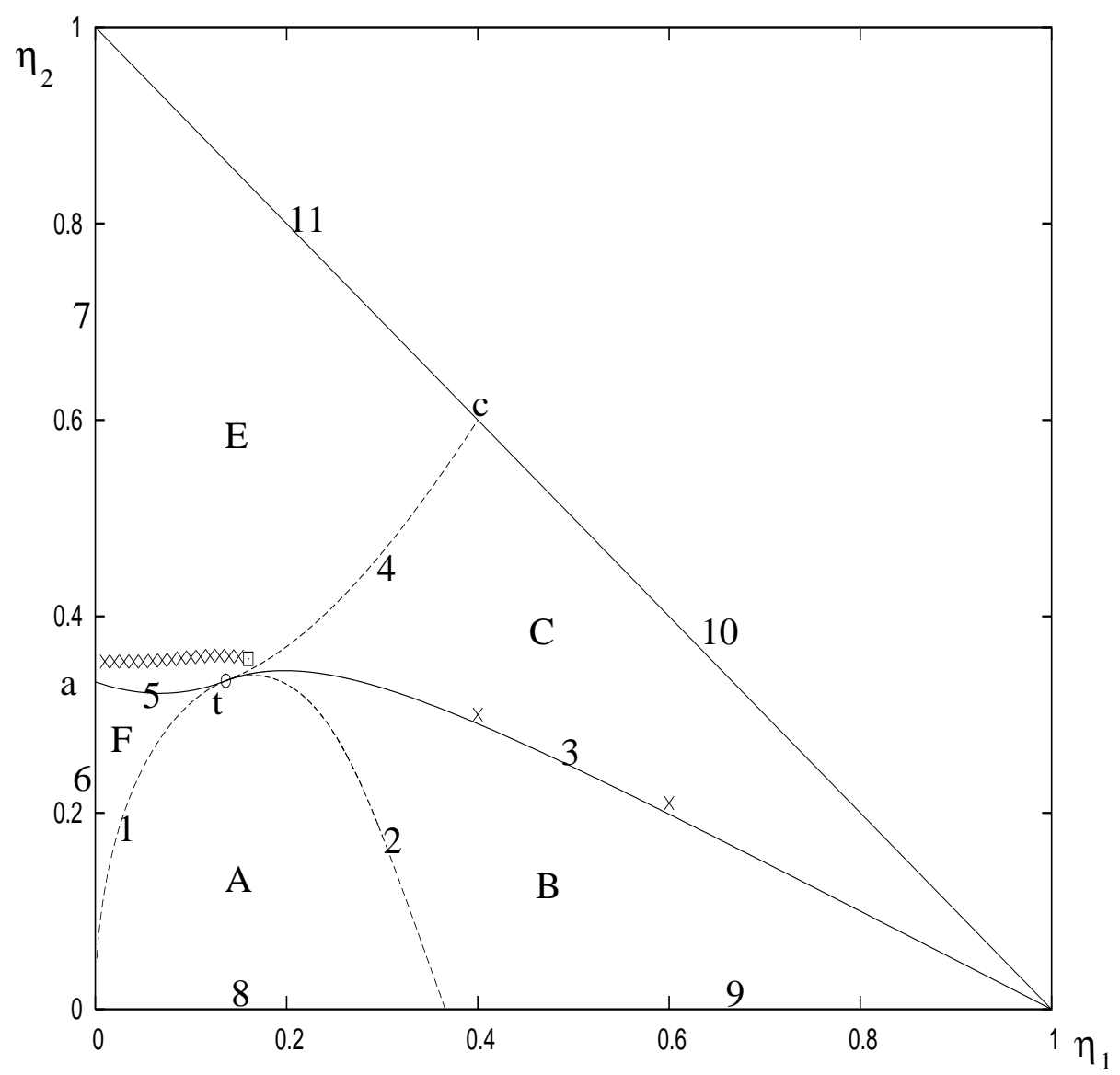

Figure 1: Phase diagram of the classical three-frequency sine-Gordon model at $\beta_{1} / \beta_{2} / \beta_{3}=$ $3 / 2 / 1, \delta_{1}=\delta_{2}=\delta_{3}=0, \mu_{1}, \mu_{3}<0$ and $\mu_{2}>0$. The crosses and the square correspond to certain quantum theory values described in Section 7 . 

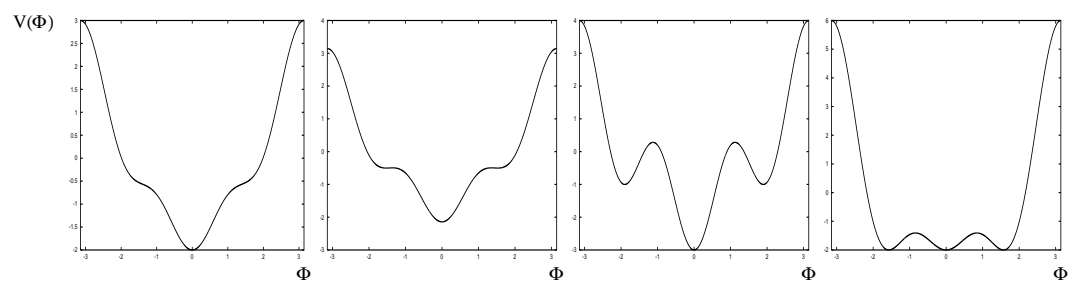

$1,6,8, \mathrm{~A}, \mathrm{~F}$

2

$\mathrm{B}$

3

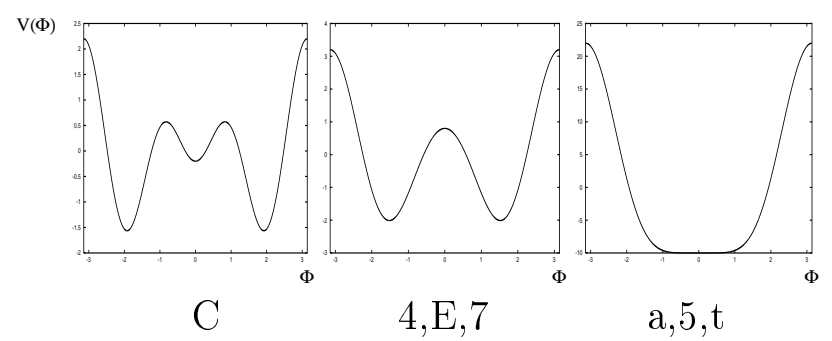

Figure 2: Characteristic shapes of the potential

\section{$4.3 n$-frequency model in the classical limit}

Let us take the $n$-frequency model with $\beta_{i}=i \beta, i=1 . . n$, and $\delta_{i}=0$. In this case there exist unique values of $\mu_{i} / \mu_{1}, i=2 . . n$ so that $V(x)$ has a single global minimum at $x=0$ and has no other local minima, and $V^{\prime \prime}(0)=0, V^{\prime \prime \prime \prime}(0)=0, \ldots, V^{(2 n)}(0)=0$ also hold. The values of $\mu_{i} / \mu_{1}$ are determined by the latter equations. The point corresponding to these values of $\mu_{i} / \mu_{1}$ is an $n$-fold multicritical point in the phase space. The neigbourhood of this multicritical point contains $m$-fold multicritical points for any integer $0<m<n$.

These statements can be proved using well known properties of analytic functions and the fact that $V$ is a trigonometric polynomial. We omit the details of the proof.

\section{Signatures of 1st and 2nd order phase transitions in finite volume}

The considerations in this section apply to quantum field theory.

The behaviour of the spectrum is governed by the $l \rightarrow 0$ limiting conformal field theory for small values of $l$, so $e_{n}(l)-e_{0}(l) \sim 1 / l$. Massive phases in infinite volume are characterized by the existence of a massgap and the behaviour $\lim _{l \rightarrow \infty}\left(e_{n}(l)-e_{0}(l)\right)=C_{n}$, where $C_{n} \geq 0$ are constants. $C_{n}=0$ if $0 \leq n \leq d$ and $C_{n}>0$ if $n>d$, if the ground state has $d$-fold degeneracy in infinite volume. In a phase with spontaneously broken symmetry the spectrum is degenerate in the $l \rightarrow \infty$ limit, in finite volume the degeneracy is lifted (at least partially) due to tunneling effects. The resulting energy split between the degenerate vacua vanishes exponentially as $l \rightarrow \infty$. 
In the critical points (in infinite volume) the massgap vanishes and the Hilbert space contains a sector that corresponds to the conformal field theory specifying the universality class of the critical point. We consider this sector in the following discussion. In finite but large volume near the critical point this sector of the theory can be regarded as the $l \rightarrow \infty$ limiting conformal theory perturbed by some irrelevant and relevant operators. The corresponding TCSA Hamiltonian takes the (generic) form

$$
H=\frac{2 \pi}{L}\left(\left(L_{0}\right)_{I R}+\left(\bar{L}_{0}\right)_{I R}-\frac{c_{I R}}{12}+\sum_{\psi} \frac{g_{\psi} L^{2-2 \Delta_{\psi}}}{(2 \pi)^{1-2 \Delta_{\psi}}} \psi(1,1)\right)
$$

where the $\psi$ are the perturbing fields. This picture gives the following volume dependence of energy levels (in the first order of conformal perturbation theory):

$$
e_{\Psi}(l)-e_{0}(l)=\frac{2 \pi}{l}\left(\Delta_{I R, \Psi}^{+}+\Delta_{I R, \Psi}^{-}\right)+\sum_{\psi} A_{\Psi}^{\psi} l^{1-2 \Delta_{\psi}},
$$

where $\Delta_{I R, \Psi}^{+}$and $\Delta_{I R, \Psi}^{-}$are the conformal weights of the state $\Psi$ in the $l \rightarrow \infty$ limiting CFT, $A_{\Psi}^{\psi}$ are constants that also depend on the particular energy eigenstate $\Psi$. The presence of irrelevant perturbations $\left(1-2 \Delta_{\psi}<-1\right)$ is due to the finiteness of the volume, whereas the presence of the relevant perturbations $\left(1-2 \Delta_{\psi}>-1\right)$ is caused by the deviation of the control parameters from the critical value and by the UV cutoff.

The location of the critical points can be determined using the criterion of vanishing massgap. A more precise method that also allows the determination of the universality class of the critical point (i.e. the $l \rightarrow \infty$ limiting CFT) is the following: We make an assumption that the critical point is in a certain universality class. This assumption predicts the set of $\psi$-s, the values of $\Delta_{I R, \Psi}^{+}$and $\Delta_{I R, \Psi}^{-}$, and the values of the $\Delta_{\psi^{-}}$in (6) . We take leading terms of the series on the r.h.s. of (66) and determine the value of the $\left(\Delta_{I R, \Psi}^{+}+\Delta_{I R, \Psi}^{-}\right)$-S and of the $A_{\Psi}^{\psi}$-S by fitting to the TCSA energy data. The magnitude of the $A_{\Psi}^{\psi}$-s corresponding to the relevant perturbations measures the deviation from the critical point, so if the assumption on the universality class is right, then by tuning the coupling constants one should be able to find a (critical) value of the control parameter at which these $A_{\Psi}^{\psi}$-S are small, the TCSA data are described well by (6) (terms from higher orders of perturbation theory can be included if necessary) in a reasonably large interval of the values of $l$, and the values of the $\left(\Delta_{I R, \Psi}^{+}+\Delta_{I R, \Psi}\right)$-s obtained from the TCSA data agree with the assumption with good precision. The interval where (6) describes the TCSA data well is called the scaling region. This region may be (and in fact is) different for different energy levels. We remark that it is also possible to make a theoretical prediction for $e_{0}(l)$, which allows to extract $c_{I R}$ from the TCSA data for $e_{0}(l)$ in principle [3]. However, experience $([3])$ shows that the accuracy of the TCSA data is not sufficient to determine $c_{I R}$ precisely in this way, so we do not attempt to extract $c_{I R}$ directly from the TCSA data in this paper. 


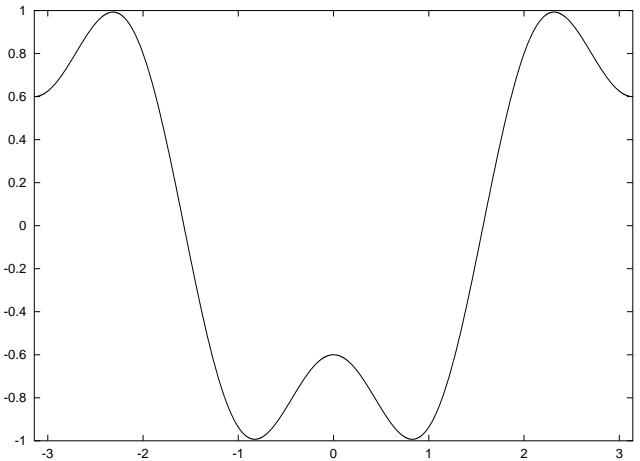

a

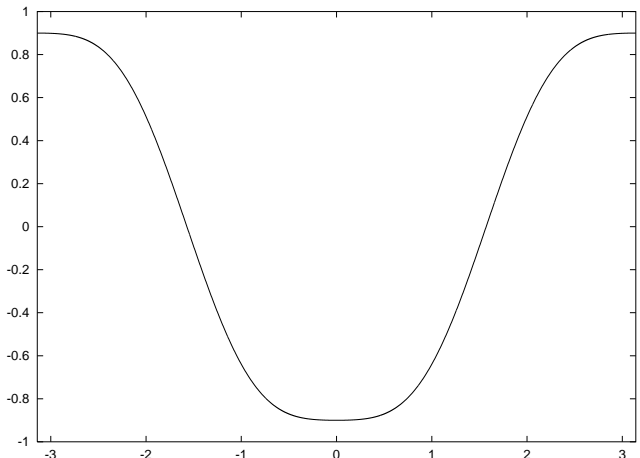

$\mathrm{b}$

Figure 3: Typical shape of $V(\Phi)$ in the broken and in the unbroken symmetry phase

In the classical case a first order phase transition occurs when the absolute minimum of the potential becomes a relative minimum and a previously relative minimum becomes absolute. In the quantum case this phase transition is characterized by the presence of "runaway" energy levels with asymptotic behaviour $e(l) \sim c l$ for large $l$ in the neighborhood of the transition point, where $c$ is a constant that tends to zero as the transition point is approached. The multiplicity of the ground state also changes as the transition point is passed if the two phases have different symmetry properties. We remark that "runaway" energy levels are present in general whenever a model has unstable vacua.

\section{The phase diagram of the two-frequency model in the case $\frac{\alpha}{\beta}=\frac{1}{3}, \delta=\frac{\pi}{3}$}

We assume that $\lambda, \mu>0$, and use the parameter

$$
\tilde{\eta}=\frac{\lambda^{x_{\beta}}}{\mu^{x_{\alpha}}+\lambda^{x_{\beta}}}
$$

instead of $\eta$ in this case to conform with $[3]$.

The classical model with $\alpha / \beta=\frac{1}{3}, \delta=\frac{\pi}{3}$ exhibits an Ising type phase transition at $\tilde{\eta}=3^{4} /\left(1+3^{4}\right)$.

Considering the quantum case we proceed along the lines of [3] in this section. The numerical nature of the TCSA makes it necessary to choose a finite number of values for $\beta$ and $l$ at which calculations are done. One should choose as large values for $l$ as possible, the $l \rightarrow \infty$ limit being of interest. However, the accuracy of TCSA decreases as $l$ grows. The accuracy can be improved by taking higher $e_{c u t}$, but this increases the size of TCSA Hamiltonian and the time needed for diagonalization. Experience shows that accuracy 
decreases for values $\beta$ near to $\sqrt{4 \pi}$ (this is the value where UV divergences appear in conformal perturbation theory) although the speed of convergence of the spectrum to the $l \rightarrow \infty$ asymptotic values increases, and the speed of convergence becomes very low for values of $\beta$ near to 0 . Taking these properties of the TCSA into consideration and following [3] we performed calculations at the values $\beta=8 \sqrt{\pi} / 7,4 \sqrt{\pi} / 3,8 \sqrt{\pi} / 5$. We also note that the accuracy of the TCSA spectra is severely decreased if $V$ has several (local) minima.

Figure 3, a and 3. b show the shape of the classical potential in the phases with broken and unbroken $\mathbb{Z}_{2}$-symmetry, respectively. Figures 4. a-4. g show TCSA spectra obtained at $\beta=4 \sqrt{\pi} / 3$ at various values of $\tilde{\eta}$. The TCSA Hilbert space had dimension 3700, the first 12 energy levels are shown in the figures. The highest values of $l$ are chosen so that the truncation error still be small (the massgap remain constant). However, the effect of truncation is perceptible in Figure 4.b for instance. It can be seen that in the domain $\tilde{\eta}<0.92$ the ground states and the first massive states are doubly degenerate. (They are triply degenerate at $\tilde{\eta}=0$.) "Runaway" energy levels (of constant slope) corresponding to the single local minimum of the potential can also be seen (especially clearly in Figure 4. b). In the domain $\tilde{\eta}>0.98$ the spectra are massive, but the ground state and the first massive state are nondegenerate. In the intermediate domain (especially for $\tilde{\eta} \sim 0.95$ ) the structure of the spectrum changes, no massgap and degeneracy can clearly be seen. We obtained similar spectra at $\beta=8 \sqrt{\pi} / 5$ and $\beta=8 \sqrt{\pi} / 7$ as well. As we did not see "runaway" energy levels that would have signaled first order phase transition in the transitional domain of $\tilde{\eta}$ we analyzed the data by looking for a second order Ising type phase transition at some critical values $\tilde{\eta}_{c}(\beta)$.

The Ising model contains three primary fields: the identity with weights $(0,0)$, the $\epsilon$ with weights $(1 / 2,1 / 2)$, and $\sigma$ with weights $(1 / 16,1 / 16)$. Since the DSG model exhibits the $\mathbb{Z}_{2}$-symmetry for all values of $\tilde{\eta}$, the $\mathbb{Z}_{2}$-odd $\sigma$ and its descendants cannot appear as perturbations in the Hamiltonian (5). The only relevant field compatible with the $\mathbb{Z}_{2}$-symmetry is $\epsilon$ (the contribution of the identity cancels in the relative energy levels). The presence of a relevant perturbation $\epsilon$ in the Hamiltonian leads to a correction $B_{\Psi}$ or $B_{\Psi}+C_{\Psi} l$ to $e_{\Psi}(l)-e_{0}(l)$. The term $C_{\Psi} l$ is of second order in perturbed CFT. The leading irrelevant perturbation (compatible with the $\mathbb{Z}_{2}$-symmetry) is the first descendant of $\epsilon$, this gives a correction $A_{\Psi} l^{-2}$ to $e_{\Psi}(l)-e_{0}(l)$ (in first order). Thus we expect that in a large but finite volume range, near $\tilde{\eta}_{c}(\beta)$, the volume dependence of the energy levels is described well by the formula

$$
e_{i}(l)-e_{0}(l)=\frac{2 \pi}{l} D_{i}+A_{i} l^{-2}+B_{i}+C_{i} l .
$$

We fitted this function to the lowest energy levels obtained by TCSA and determined the "best" $\tilde{\eta}_{c}(\beta)$ value by tuning $\tilde{\eta}$ in the transition region and looking for whether $e_{2}(l)-e_{0}(l)$ continues to decrease along the complete $l$ range $\left(\lim _{l \rightarrow \infty}\left(e_{2}(l)-e_{0}(l)\right)=0\right.$ only at $\left.\tilde{\eta}_{c}(\beta)\right)$, and $B_{i}$ and $C_{i}$ are as small as possible. The result is shown in Table 11. The fitting was 


\begin{tabular}{|c|c|c|c|c|}
\hline State & $D_{i}$ & $A_{i}$ & $B_{i}$ & $C_{i}$ \\
\hline$i=1$ & $0.138 \pm 0.0005$ & $-2.0 \pm 0.02$ & $-0.0046 \pm 0.0001$ & $2.98 \cdot 10^{-5} \pm 9 \cdot 10^{-7}$ \\
\hline$i=2$ & $1.00 \pm 0.01$ & $-74 \pm 2$ & $0.004 \pm 0.001$ & $9 \cdot 10^{-6} \pm 4 \cdot 10^{-6}$ \\
\hline
\end{tabular}

$$
\beta=8 \sqrt{\pi} / 5, \tilde{\eta}=0.944
$$

\begin{tabular}{|c|c|c|c|c|}
\hline State & $D_{i}$ & $A_{i}$ & $B_{i}$ & $C_{i}$ \\
\hline$i=1$ & $0.125 \pm 0.001$ & $-2.63 \pm 0.025$ & $-0.0002 \pm 0.0001$ & $3 \cdot 10^{-7} \pm 1 \cdot 10^{-6}$ \\
\hline$i=2$ & $1.04 \pm 0.02$ & $-152 \pm 6$ & $0.0014 \pm 0.0009$ & $-1.5 \cdot 10^{-5} \pm 2 \cdot 10^{-6}$ \\
\hline
\end{tabular}

$$
\beta=4 \sqrt{\pi} / 3, \tilde{\eta}=0.955
$$

\begin{tabular}{|c|c|c|c|c|}
\hline State & $D_{i}$ & $A_{i}$ & $B_{i}$ & $C_{i}$ \\
\hline$i=1$ & $0.125 \pm 0.001$ & $-4.4 \pm 0.1$ & $-0.0009 \pm 0.0001$ & $4 \cdot 10^{-7} \pm 5 \cdot 10^{-7}$ \\
\hline$i=2$ & $1.00 \pm 0.02$ & $-252 \pm 9$ & $0.0002 \pm 0.0004$ & $4.3 \cdot 10^{-6} \pm 6 \cdot 10^{-7}$ \\
\hline
\end{tabular}

$$
\beta=8 \sqrt{\pi} / 7, \tilde{\eta}=0.961
$$

Table 1: The results of fitting (7) to the first two excited levels for various values of $\beta$ at the estimated critical value of $\tilde{\eta}$

done in the volume ranges $l=10-105, l=55-105 ; l=10-140, l=100-190$; $l=20-200, l=150-390$. The errors presented come from the fitting process and do not contain the truncation errors which are generally much larger.

The first two energy levels above the ground state correspond to the operators $\sigma$ and $\epsilon$ in the Ising model. These operators have conformal weights $\Delta^{ \pm}=1 / 16$ and $\Delta^{ \pm}=1 / 2$, so the exact values for $D_{1}$ and $D_{2}$ are

$$
D_{1}=0.125, \quad D_{2}=1 .
$$

The results of the fits agree quite well with this prediction.

The TCSA data obtained at the estimated values of $\tilde{\eta}_{c}$ using a truncated space with dimension 4800, 5300 and 5100 are shown in Figure 5 . These figures show energy levels multiplied by $l /(2 \pi)$ as functions of $l$. The constant lines corresponding to the Ising model values of $D_{i}$ are also shown in these figures.

To summarize, evaluating the TCSA data we found that the phase transition is second order and Ising type at the values of $\beta$ chosen. This statement is true up to the precision of the TCSA data, which is about $10^{-2}-10^{-3}$ for the values of the volume used. We remark that it is not possible to distinguish a second order transition from a (very) weakly first order transition by TCSA. 


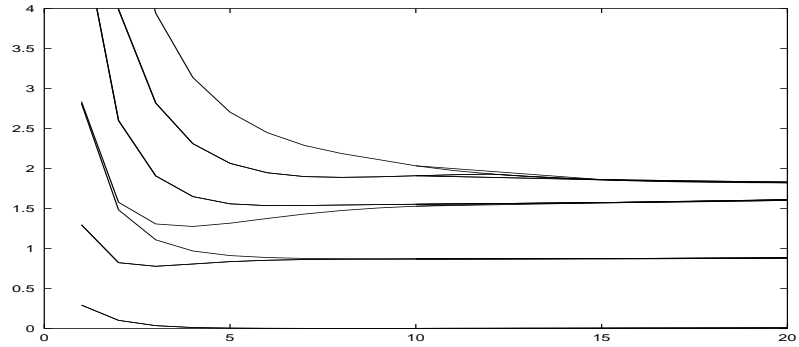

a: $\tilde{\eta}=0$

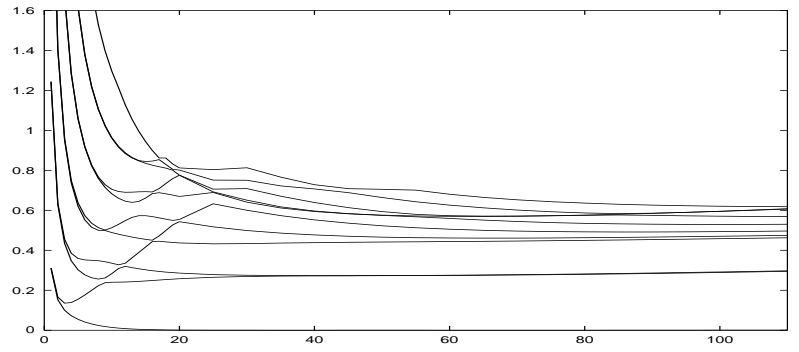

c: $\tilde{\eta}=0.7$

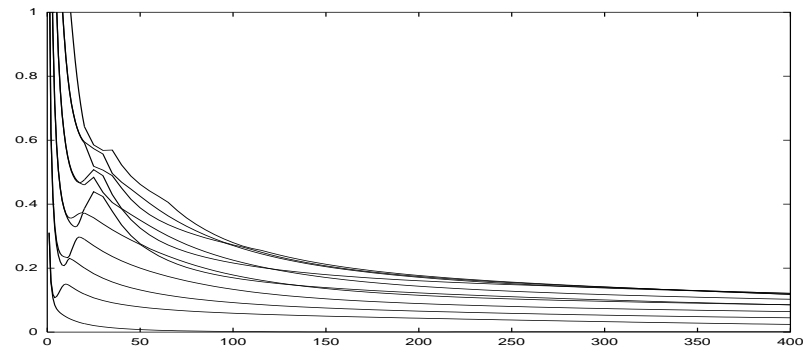

e: $\tilde{\eta}=0.94$

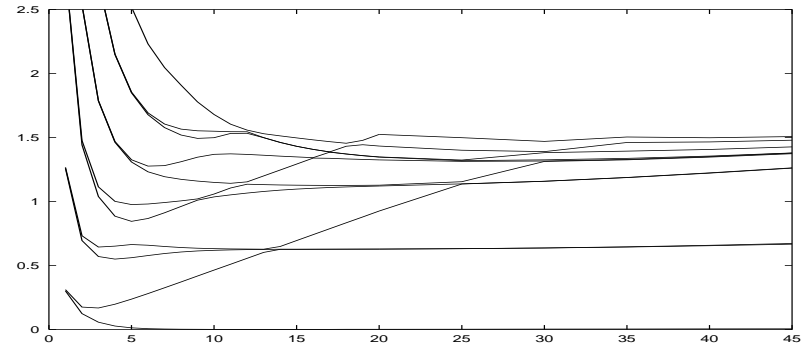

$\mathrm{b}: \tilde{\eta}=0.3$

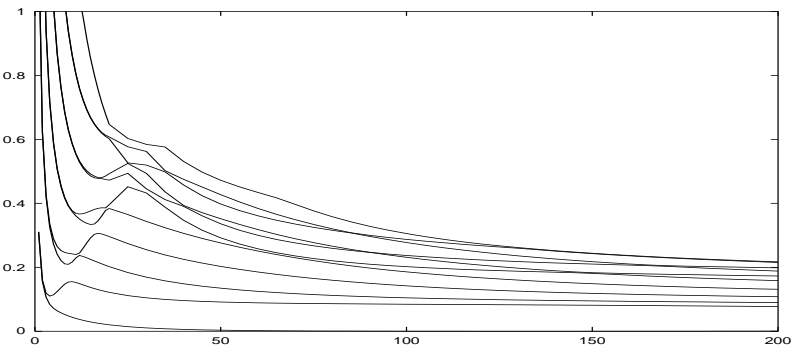

$\mathrm{d}: \tilde{\eta}=0.92$

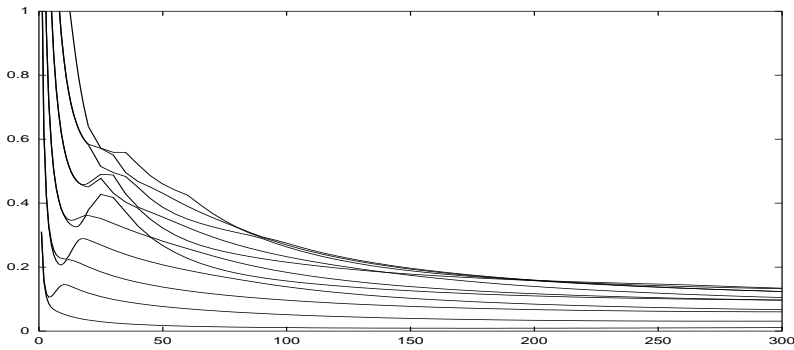

$\mathrm{f}: \tilde{\eta}=0.96$

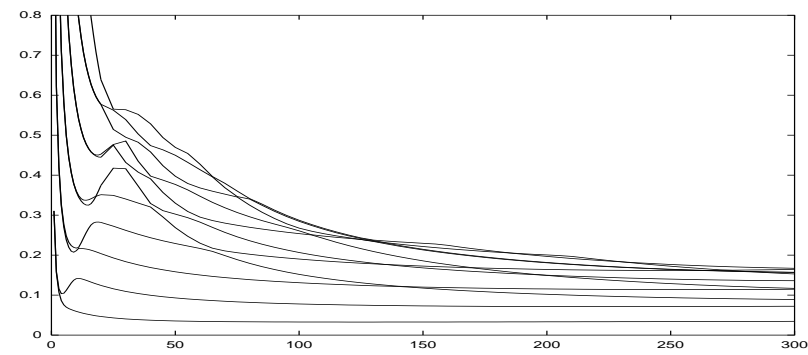

$\mathrm{g}: \tilde{\eta}=0.98$

Figure 4: Change of the spectrum as $\tilde{\eta}$ varies from 0 to 1 at $\beta=4 \sqrt{\pi} / 3$. The first 12 energy levels (including the ground level) relative to the ground level are shown as functions of $l$. 

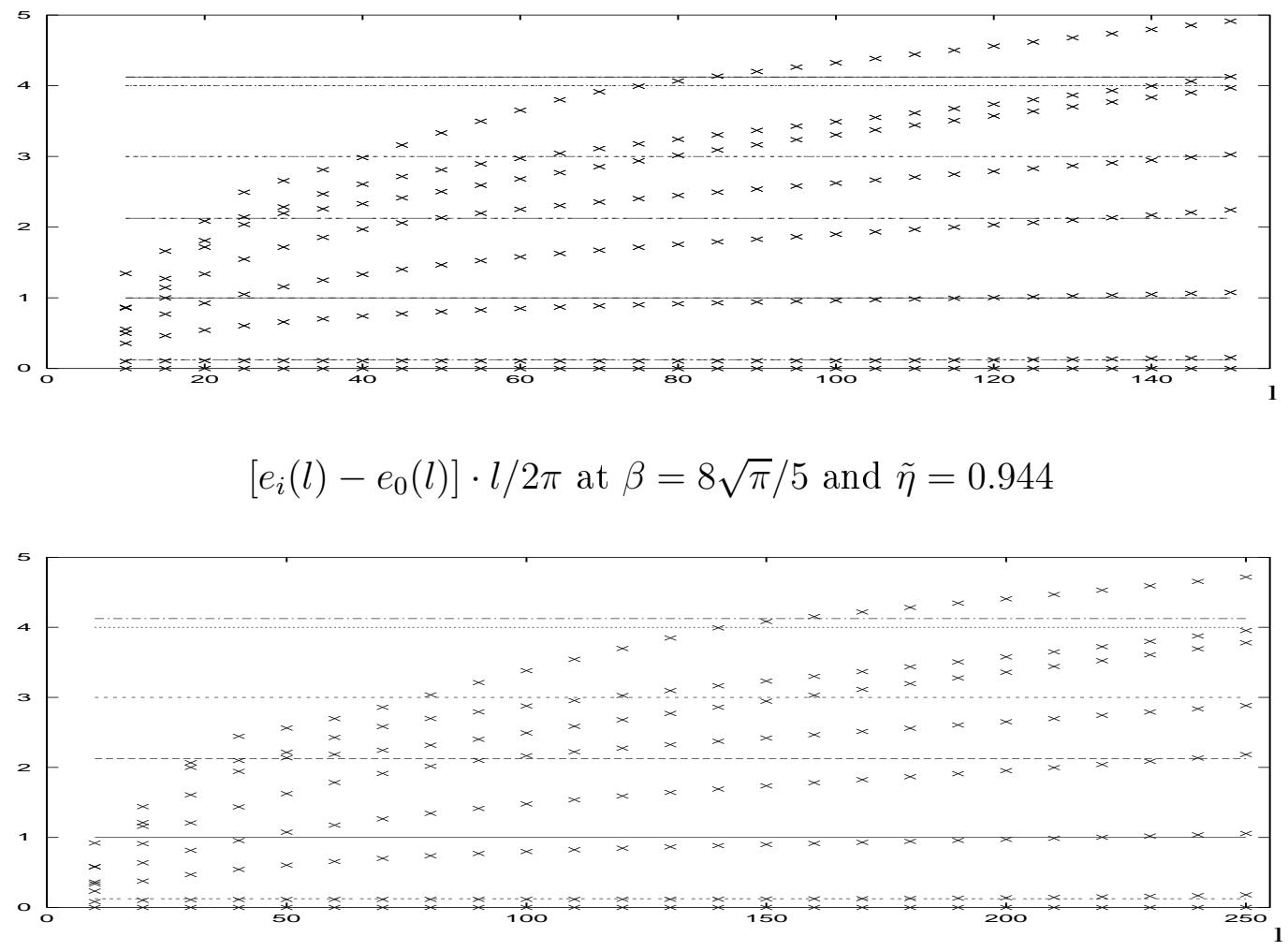

$$
\left[e_{i}(l)-e_{0}(l)\right] \cdot l / 2 \pi \text { at } \beta=4 \sqrt{\pi} / 3 \text { and } \tilde{\eta}=0.955
$$

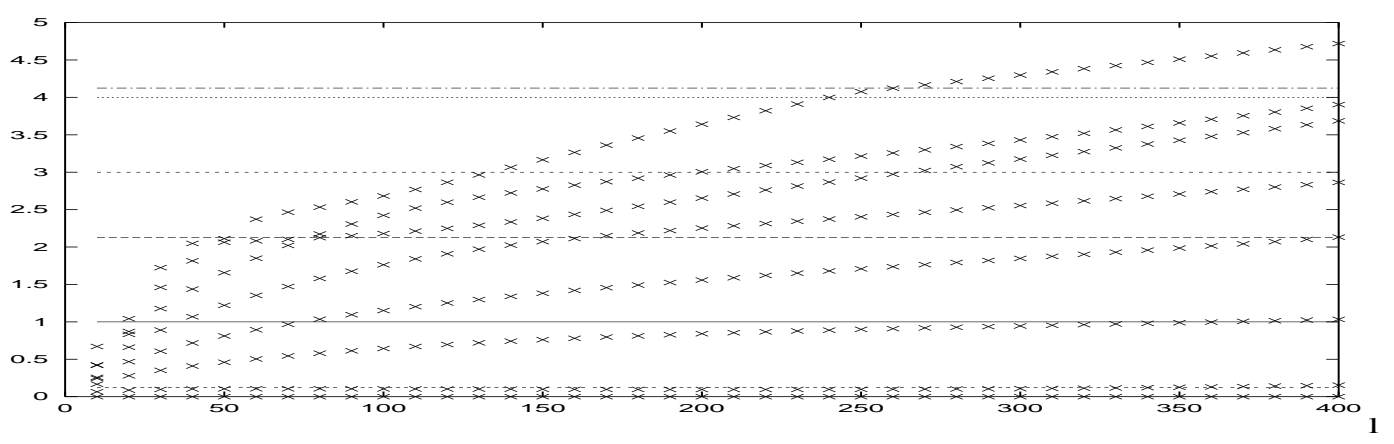

$$
\left[e_{i}(l)-e_{0}(l)\right] \cdot l / 2 \pi \text { at } \beta=8 \sqrt{\pi} / 7 \text { and } \tilde{\eta}=0.961
$$

Figure 5: TCSA spectra as functions of $l$ at the estimated critical values of $\tilde{\eta}$ 


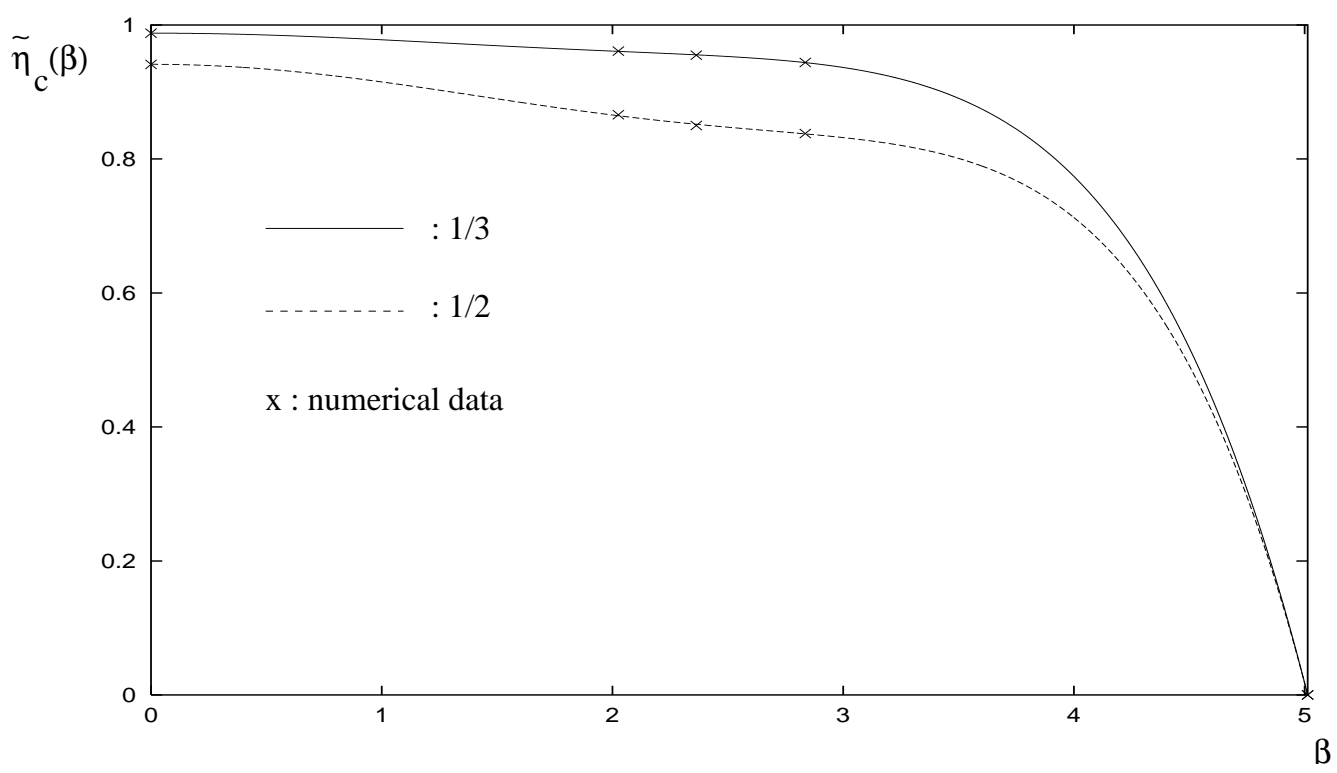

Figure 6: The phase diagram of the two-frequency model at $\alpha / \beta=1 / 2$ and $\alpha / \beta=1 / 3$

For values of $\beta$ near 0 we expect second order phase transition, because the model is semi-classical in this region and so the correction to the classical potential in the effective potential is expected to be small.

A correction to the classical potential in the effective potential of frequency $2 \beta / 3$ is possible in principle. A correction with this frequency is - unlike in the case of $\alpha / \beta=$ $1 / 2$ - relevant for any values of $\beta$, and it can be verified by elementary calculation that it may change the order of the transition outside the semiclassical region if its coefficient is sufficiently large (see also [2, 3]). However, the accuracy of TCSA did not allow us to perform calculations at values about $\beta^{2}>8 \pi / 3$ and to check the nature of phase transition in this domain.

The phase diagram in the $(\beta, \tilde{\eta})$ plane based on the data obtained by TCSA can be seen in Figure 6. We took into consideration that $\tilde{\eta}_{c}(0)=3^{4} /\left(1+3^{4}\right) \approx 0.988$ is exactly known $\beta=0$ being the classical limit, and at $\beta=\sqrt{8 \pi}$ the term with frequency $\beta$ in the potential becomes irrelevant and thus for $\beta \rightarrow \sqrt{8 \pi}$ the other term and so the symmetric phase is expected to dominate, so $\lim _{\beta \rightarrow \sqrt{8 \pi}} \tilde{\eta}_{c}(\beta)=0$. The figure shows the three values of $\tilde{\eta}_{c}$ obtained from the TCSA data and the two values at $\beta=0$ and $\beta=\sqrt{8 \pi}$. The continuous line is obtained by fitting an even polynomial (note that $\tilde{\eta}_{c}(\beta)=\tilde{\eta}_{c}(-\beta)$ ) to these values and is shown in order to get an idea of the phase transition line. The model is in the symmetric phase above the line and in the phase with broken symmetry below the line. The data obtained by [3] for the $\alpha / \beta=1 / 2$ case are also shown, the dashed line is fitted to these data. 


\section{Phase diagram of the three-frequency model}

We take the same values of the parameters of (41) as in section 4.2. namely $\beta_{1}=\beta, \beta_{2}=$ $\frac{2}{3} \beta, \beta_{3}=\frac{1}{3} \beta, \delta_{2}=\delta_{3}=0, \mu_{1}, \mu_{3}<0, \mu_{2}>0$, and investigate the quantum model in this case.

\subsection{The tricritical point}

The tricritical Ising model contains 6 primary fields with the following conformal weights:

$$
(0,0),\left(\frac{1}{10}, \frac{1}{10}\right),\left(\frac{3}{5}, \frac{3}{5}\right),\left(\frac{3}{2}, \frac{3}{2}\right),
$$

and

$$
\left(\frac{3}{80}, \frac{3}{80}\right),\left(\frac{7}{16}, \frac{7}{16}\right) .
$$

The fields corresponding to (8) are even and those corresponding to (9) are odd with respect to the parity, so only the fields corresponding to (8) and their descendants can contribute to the Hamiltonian (5). Thus the volume dependence of the energy levels near the tricritical point should be described well for large $l$ by

$$
e_{\Psi}(l)-e_{0}(l)=\frac{2 \pi}{l}\left(\Delta_{I R, \Psi}^{+}+\Delta_{I R, \Psi}\right)+A_{\Psi} l^{-0.2}+B_{\Psi} l^{0.8}+\ldots,
$$

where only the leading terms are kept. Searching for the tricritical point we fitted the function

$$
\frac{2 \pi}{l} D_{i}+A_{i} l^{-0.2}+B_{i} l^{0.8}
$$

to the data obtained by TCSA for $e_{i}(l)-e_{0}(l)$ near the estimated location of the tricritical point. Best fits are shown in Table 2. (The errors presented come from the fitting process and do not contain the truncation errors which are generally much larger.) The fitting was done in the volume ranges $l=50-230, l=110-230$, the dimension of the truncated Hilbert space was 13600 . The results of the fitting support the existence of a tricritical point located (approximately) at $\eta_{1}=0.163, \eta_{2}=0.3518$. The exact values of $D_{1}$ and $D_{2}$ in the tricritical Ising model are

$$
D_{1}=0.075, \quad D_{2}=0.2 .
$$

The numerical results agree quite well with this prediction. The TCSA spectrum obtained at the tricritical point is shown in Figure 7 . The values of $D_{i}$ predicted by the tricritical Ising model are also shown in the figure. The dashed lines and + signs are used for odd parity states, the continuous lines and $\times$ signs are used for even parity states. 


\begin{tabular}{|c|c|c|c|}
\hline State & $D_{i}$ & $A_{i}$ & $B_{i}$ \\
\hline$i=1$ & $0.074 \pm 0.004$ & $-0.0060 \pm 0.001$ & $2.8 \cdot 10^{-5} \pm 5 \cdot 10^{-6}$ \\
\hline$i=2$ & $0.196 \pm 0.01$ & $-0.006 \pm 0.002$ & $2.4 \cdot 10^{-5} \pm 6 \cdot 10^{-6}$ \\
\hline
\end{tabular}

$$
\beta=8 \sqrt{\pi} / 7, \eta_{1}=0.163, \eta_{2}=0.3518
$$

Table 2: The results of fitting (10) to the first two excited levels in the estimated tricritical point

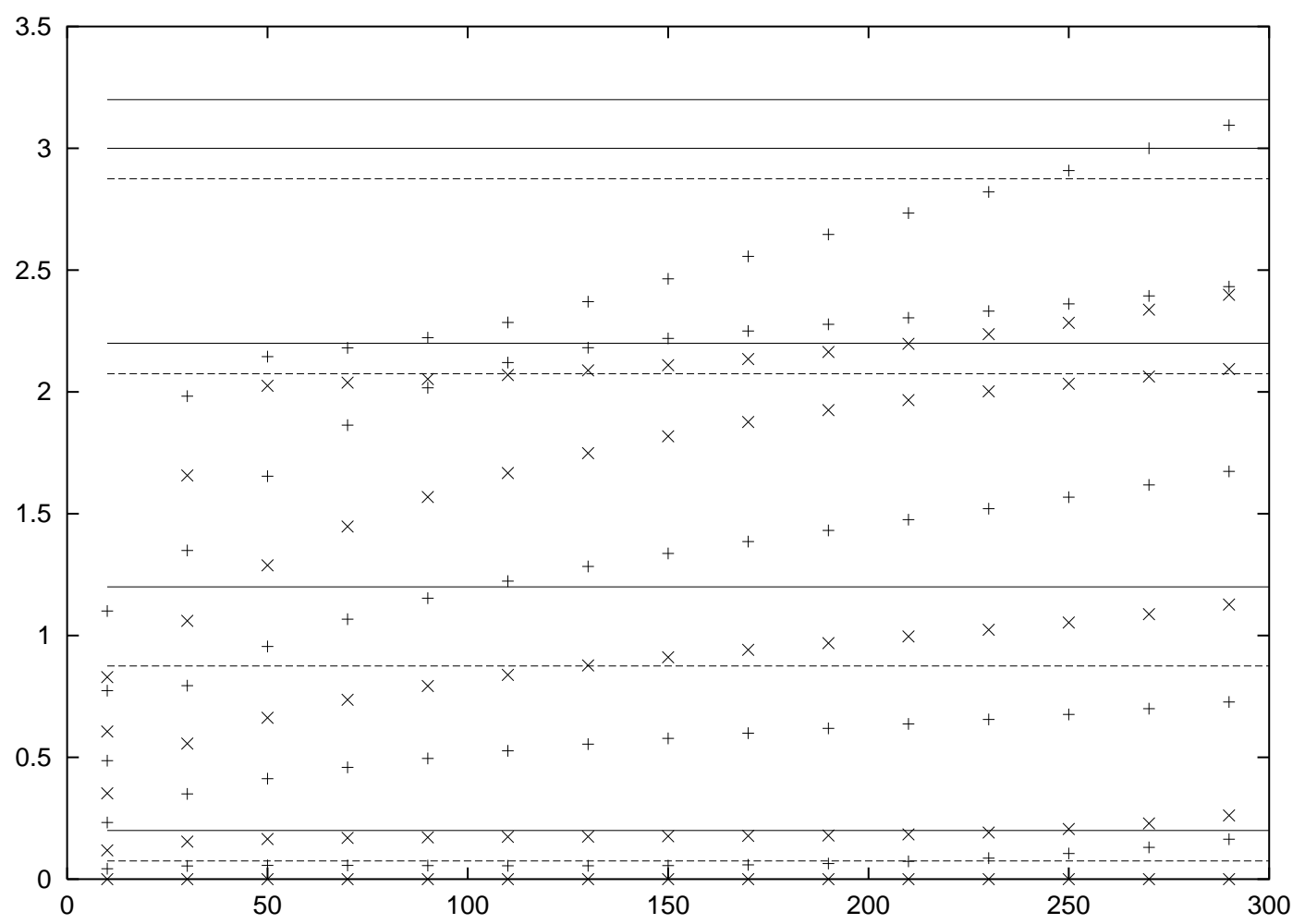

Figure 7: $\left[e_{i}(l)-e_{0}(l)\right] \cdot l / 2 \pi$ as functions of $l$ obtained by TCSA at $\beta=8 \sqrt{\pi} / 7, \eta_{1}=0.163$, $\eta_{2}=0.3518$ 


\begin{tabular}{|c|c|c|c|c|c|c|c|}
\hline$\eta_{1}$ & $\eta_{2}$ & $\eta_{1}$ & $\eta_{2}$ & $\eta_{1}$ & $\eta_{2}$ & $\eta_{1}$ & $\eta_{2}$ \\
\hline 0.01 & 0.354 & 0.06 & 0.355 & 0.11 & 0.359 & 0.16 & 0.357 \\
\hline 0.02 & 0.354 & 0.07 & 0.3555 & 0.12 & 0.36 & 0.163 & 0.3565 \\
\hline 0.03 & 0.354 & 0.08 & 0.356 & 0.13 & 0.36 & & \\
\hline 0.04 & 0.354 & 0.09 & 0.3575 & 0.14 & 0.3595 & & \\
\hline 0.05 & 0.354 & 0.1 & 0.3585 & 0.15 & 0.359 & & \\
\hline
\end{tabular}

Table 3: Points of the critical line found by TCSA

We used a modified version of the TCSA code exploiting the $\mathbb{Z}_{2}$-symmetry of the model by taking even and odd basis vectors and taking the Hamiltonian on the even and odd subspaces separately, which reduces the total time needed for diagonalization and thus allows to take higher $e_{c u t}$ values.

The TCSA data for the first two excited states fit quite well to the prediction of the tricritical Ising model, the energy levels of the next two excited states also show correspondence with the prediction. Clear correspondence cannot be seen for higher levels.

\subsection{The critical line}

The points of the critical line we found using TCSA are listed in Table 3. The value of $\eta_{1}$ was chosen and fixed in advance, and then $\eta_{2}$ was estimated in the same way as described in Section 6. These points are also marked in Figure 1 by crosses. The dimension of the truncated Hilbert space was 10269 in these calculations, which corresponded to $e_{\text {cut }}=17$. The value of $\beta$ was $8 \sqrt{\pi} / 5$. Figures 8 , a-1 show the TCSA spectra (especially the lowest lying energy levels) obtained in these points as well as the values of $D_{i}$ corresponding to both the critical and the tricritical Ising model. Crosses are used for odd parity states, squares are used for even parity states. It can be seen that moving on the critical line in the phase space towards the tricritical endpoint the finite volume spectrum changes continuously. In the $\eta_{1}<0.11$ domain the spectra (especially the first two levels) correspond clearly to phase transitions in the Ising universality class. At $\eta_{1}=0.11$ the first excited level already appears to correspond to the prediction of the tricritical Ising model, whereas the second excited level still has the behaviour predicted by the Ising model. It would be very interesting to know the large volume behaviour (and infinite volume limit) of the first excited level, but the precision of TCSA does not allow to determine it. What we can see is that there is no sign in the TCSA data that the first excited level follows the predictions of the Ising model in the large volume limit. In the domain $0.11 \leq \eta_{1} \leq 0.16$ there is a spectacular rearrangement of the higher energy levels (already observable in the $\eta_{1}<0.11$ domain), and at $\eta_{1}=0.16$ the second excited level also appears to correspond to the prediction of the tricritical Ising model. We regard therefore the point $\eta_{1}=0.16$, 
$\eta_{2}=0.357$ to be the tricritical endpoint of the critical line, this point is marked by a square in Figure 1. (We did not aspire to determine the value of $\eta_{1}$ more precisely for this value of $\beta$.) Each figure shows the spectrum in the volume interval $l=0 . .200$, and for the large values $l \approx 200$ the truncation error is always conspicuous.

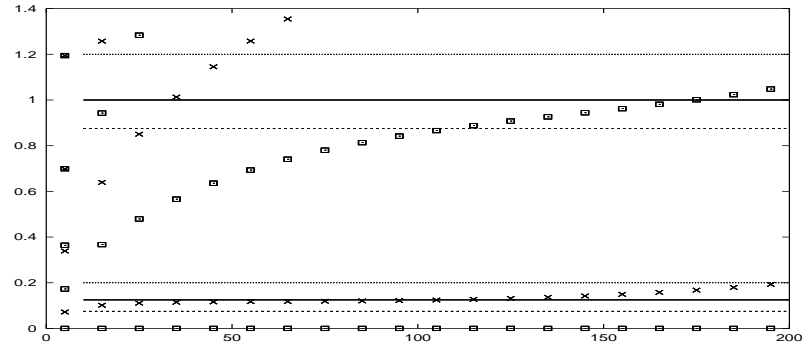

$\mathrm{a}: \eta_{1}=0.01$

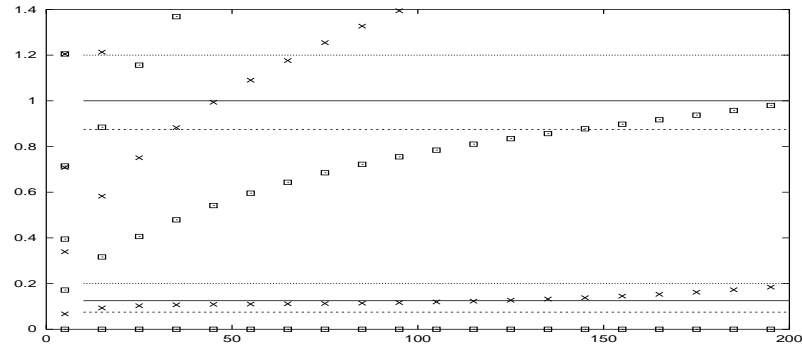

c: $\eta_{1}=0.08$

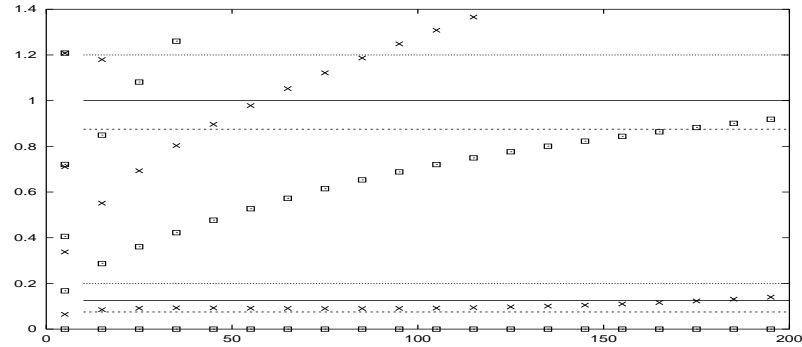

$\mathrm{e}: \eta_{1}=0.10$

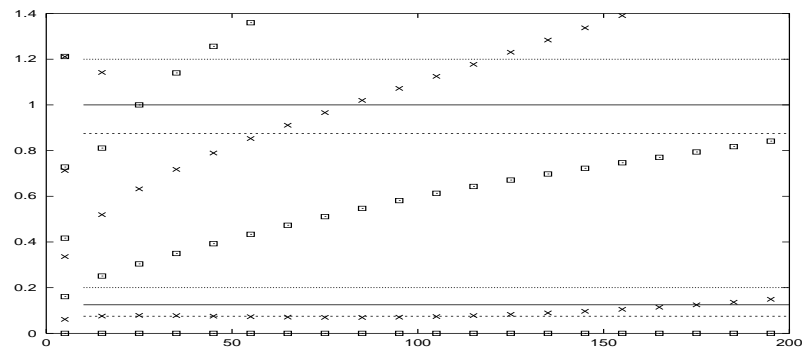

$\mathrm{g}: \eta_{1}=0.12$

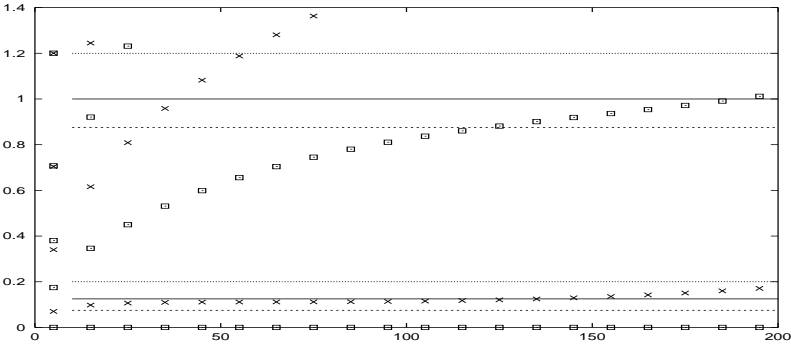

b: $\eta_{1}=0.05$

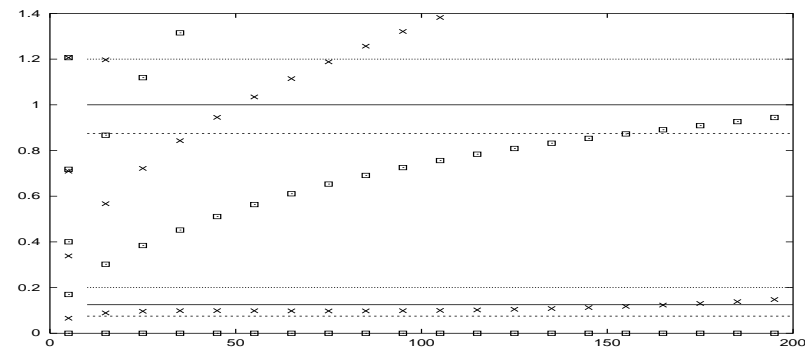

$\mathrm{d}: \eta_{1}=0.09$

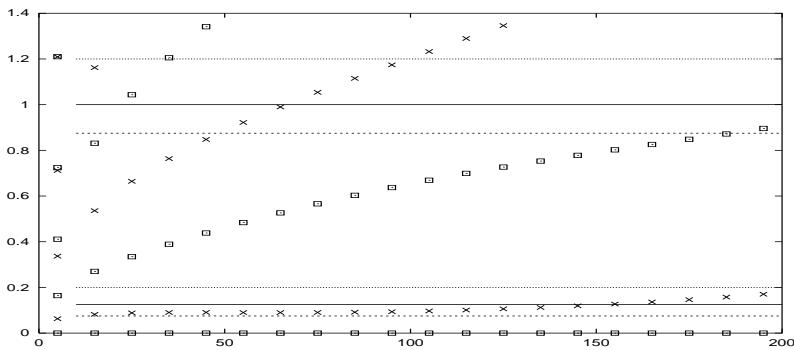

f: $\eta_{1}=0.11$

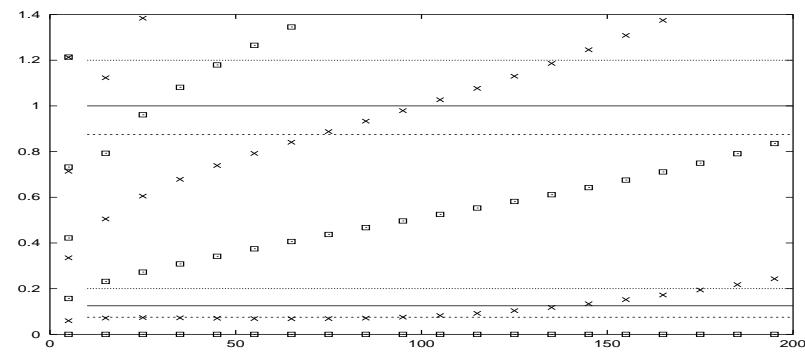

$\mathrm{h}: \eta_{1}=0.13$ 


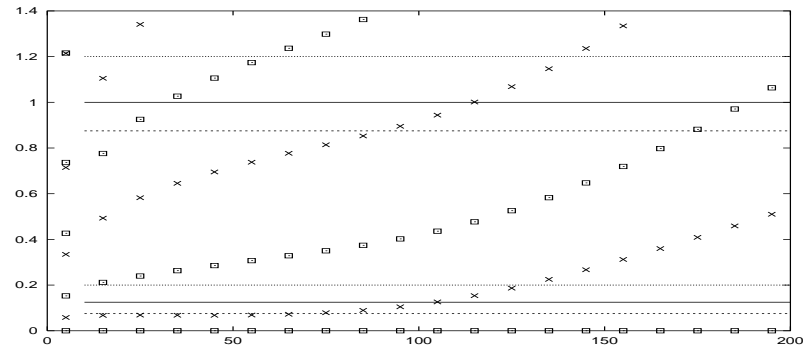

i: $\eta_{1}=0.14$

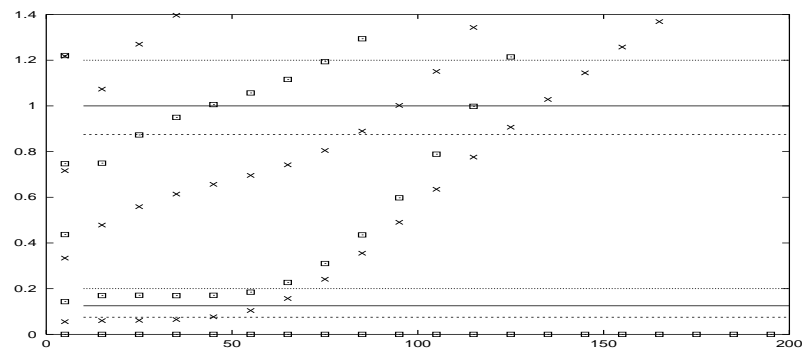

$\mathrm{k}: \eta_{1}=0.16$

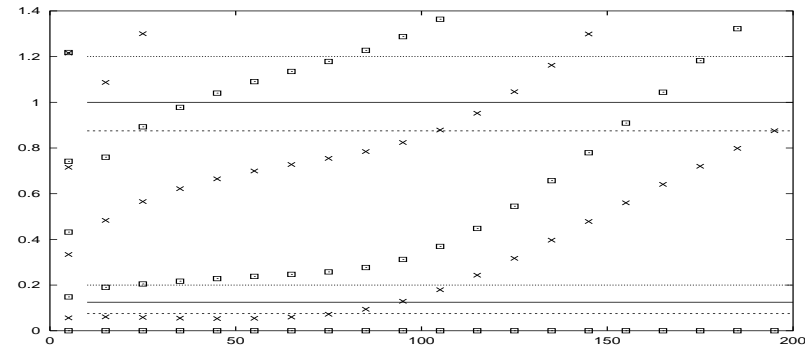

$\mathrm{j}: \eta_{1}=0.15$

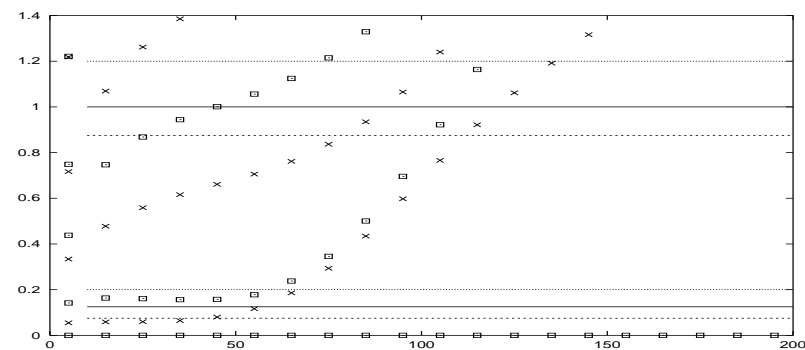

l: $\eta_{1}=0.163$

Figure 8: $\left[e_{i}(l)-e_{0}(l)\right] \cdot l / 2 \pi$ as functions of $l$ obtained by TCSA at $\beta=8 \sqrt{\pi} / 5$ and at various points $\left(\eta_{1}, \eta_{2}\right)$ lying on the critical line, the predictions of the critical Ising model (continuous horizontal lines) for $D_{i}$, the predictions of the tricritical Ising model (dashed horizontal lines) for $D_{i}$

Moreover, the value of $l$ where the truncation errors become large gets smaller and smaller as the tricritical point is approached, which corresponds to the fact that a tricritical point as renormalization group fixed point is more repelling than an Ising type one. Increasing $\eta_{1}$ further the behaviour corresponding to the tricritical point would rapidly disappear from the finite volume spectrum.

\subsection{The line of first order transition}

Figures 9 a-j show TCSA spectra obtained at $\eta_{1}=0.6$ and at various values of $\eta_{2}$ between 0.17 and 0.30 . The energy levels are shown as compared to the lowest level. The dimension of the truncated Hilbert space was 6597 in these calculations, which corresponded to $e_{c u t}=16$. The value of $\beta$ was $8 \sqrt{\pi} / 5$. Dashed lines are used for odd parity levels and continuous lines for even parity levels.

At $\eta_{2}=0.17$ (and also for $\eta_{2}<0.17$ ) the ground state is unique for all values of $l$, whereas doubly degenerate runaway levels can also be seen. At $\eta_{2}=0.19$, however, the ground level is doubly degenerate for small values of the volume but becomes nondegenerate in large volume, 


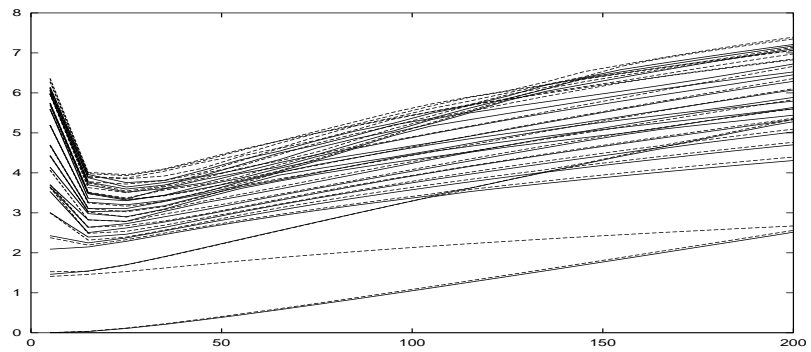

a: $\eta_{2}=0.17$

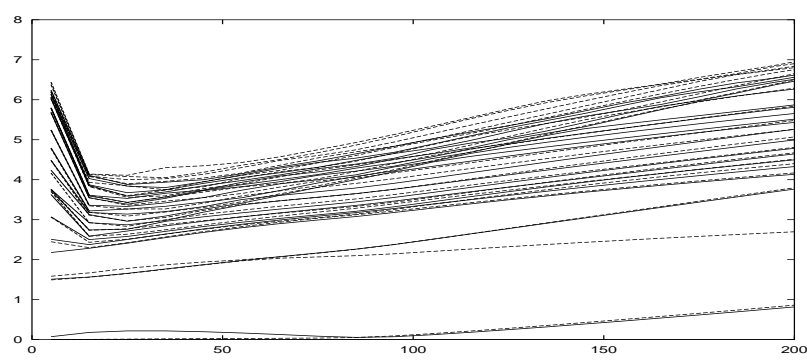

c: $\eta_{2}=0.19$

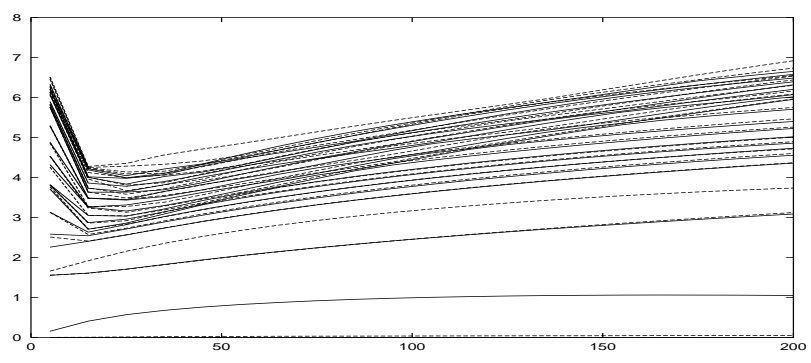

e: $\eta_{2}=0.21$

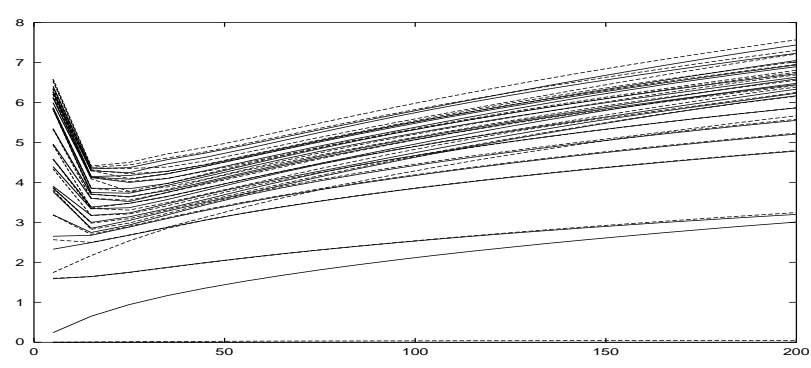

g: $\eta_{2}=0.23$

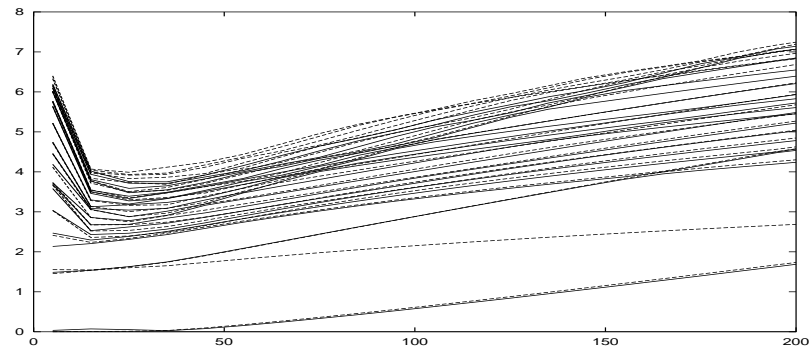

b: $\eta_{2}=0.18$

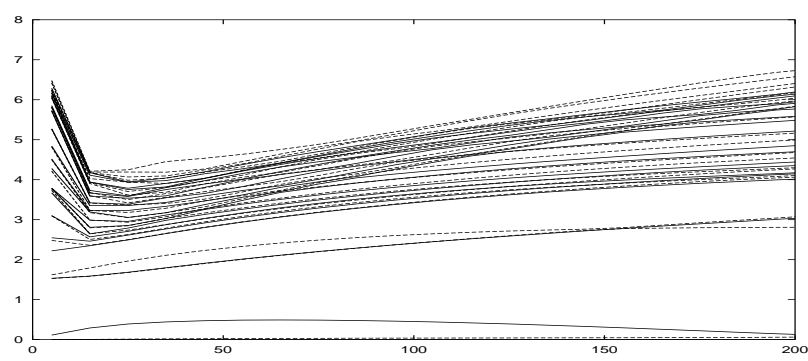

$\mathrm{d}: \eta_{2}=0.20$
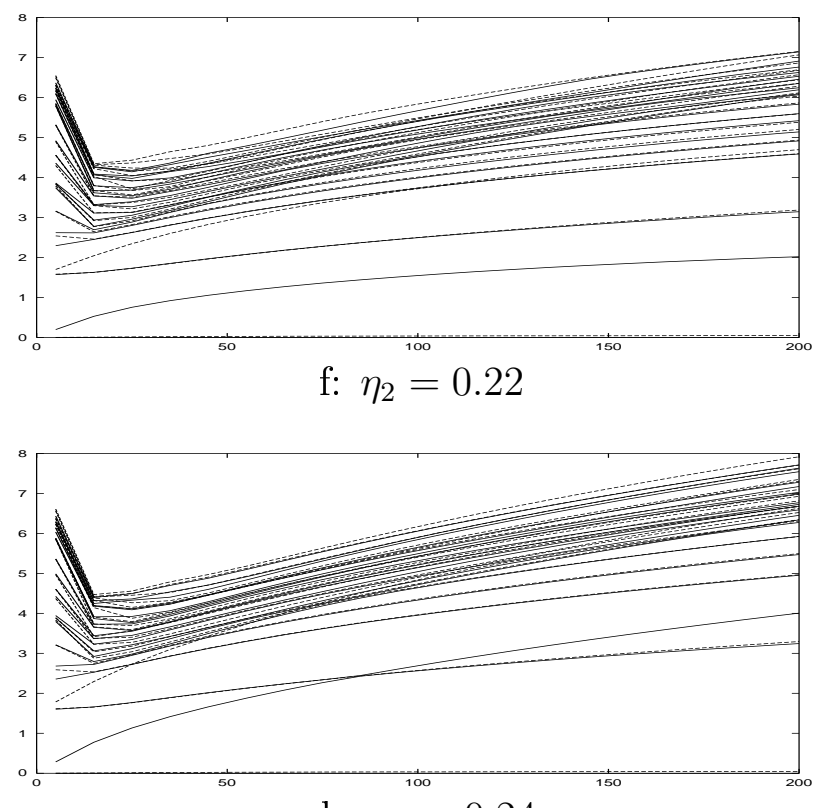

h: $\eta_{2}=0.24$ 

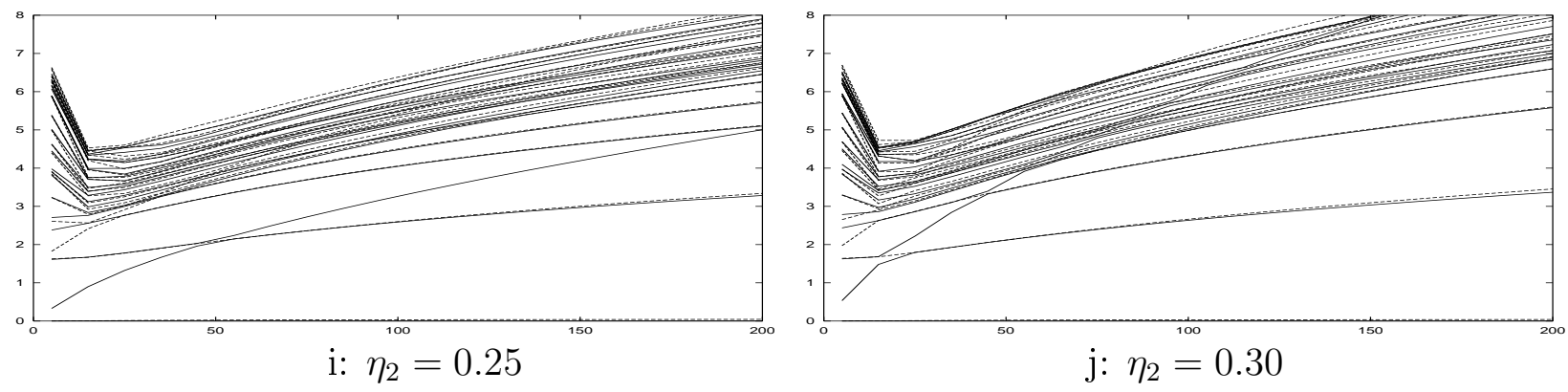

Figure 9: $\left[e_{i}(l)-e_{0}(l)\right], i=0 . .19$ as functions of $l$ obtained by TCSA at $\beta=8 \sqrt{\pi} / 5$, $\eta_{1}=0.6$ and at various values of $\eta_{2}$

i.e. there is a value of $l$ where the doubly degenerate runaway level and the single level cross each other. The slope of the runaway levels become smaller and smaller as $\eta_{2}$ is increased, and the crossing point also moves towards larger and larger values. At $\eta_{2}=0.3$ the ground state is already doubly degenerate for all values of $l$ and nondegenerate runaway levels are present. These features indicate that a first order phase transition occurs at an intermediate value of $\eta_{2}$. The behaviour of the finite volume spectra around the transition point seen in the figures implies that a precise determination of the transition point from the TCSA data in a direct way would require precise data for large values of $l$. For this reason we did not aspire to find many points of the line of first order phase transition, we did TCSA calculations only at $\eta_{1}=0.4$ and $\eta_{1}=0.6$, and we roughly estimated the location of the first order phase transition at these values. Our estimation based on the TCSA data are $\eta_{2}=0.30$ and $\eta_{2}=0.21$. These points are also marked in Figure 1 by crosses. The Figures $9 \mathrm{a}-\mathrm{j}$ show the first 20 levels in the domain $l=0 . .200$. Unfortunately the truncation effect is large in most of this domain of $l$, however we expect that those qualitative features of the spectra which we allude to are correct.

\section{Conclusions}

In this work we investigated selected special cases of the multi-frequency sine-Gordon theory, especially the structure of their phase space, continuing the work begun in [3]. Concerning the classical limit we found that the only possible phase transition in the (rational) two-frequency case is a second order Ising-type transition. The three-frequency model has some new qualitative features compared to the two-frequency model: a tricritical point which is at the end of a critical line can also be found, and first order transition is possible as well. The phase space of the $n$-frequency model contains $n$-fold critical points, otherwise we do not expect new qualitative features compared to the three-frequency model. 
The numerical (TCSA) calculations in the quantum case yielded the following results: we found the same type of phase transitions as in the classical case, in particular we were able to determine the second order Ising nature and the location of the phase transition in the two-frequency model with good precision. Quantum corrections did not alter the nature of the phase transition. The accuracy of the TCSA also allowed us to find the tricritical point in the three-frequency case, which we regard the main result of the paper. This demanded much more numerical work than the two-frequency model for the following reasons: the two-frequency model has only one-dimensional phase space, whereas the phase space is two-dimensional in the three-frequency model; and the tricritical point as a renormalization group fixed point is more repelling than the Ising-type critical point, so we had to take larger truncated space. Furthermore, we found several points of the critical line and observed how the TCSA spectrum changes as the tricritical endpoint is approached. It would be interesting to investigate this with considerably better precision. Although the focus was mainly on the tricritical point, we also investigated the first order phase transition in the three-frequency model and we found that the first order nature can be established by TCSA, but the location of the transition can be determined much less accurately than that of the second-order transition. We expect that multicritical points in the universality classes of further elements of the discrete unitary series could be found in the higher-frequency models, but increasing accuracy is needed, because these multicritical points are more and more repelling. Finally, we remark that the quantum corrections did not alter the nature of the phase transitions in any of the cases we investigated, and in the classical and quantum theory the location of transition points is almost the same. The investigation of the multi-frequency model with irrational frequency ratios is still an open problem. We also remark that the particle content of the multi-frequency sine-Gordon model could also be investigated by the method of semiclassical quantization [6].

\section{Acknowledgments}

I would like to thank L. Palla, Z. Bajnok, G. Takács and F. Wágner for useful discussions and for their TCSA program. I would also like to thank the Mathematics Department, King's College London, and especially Gerard Watts and Andreas Recknagel for their kind hospitality.

This work was partially supported by the Hungarian fund OTKA T037674. I also thank the EU Research Training Network "EUCLID", contract HPRN-CT-2002-00325, for support at various times. I also acknowledge the support by the EU Marie Curie Training Site MCFH-2001-00296 "Strings, Branes and Boundary Conformal Field Theory" at King's College London. 


\section{References}

[1] G. Delfino, G. Mussardo: Non-integrable aspects of the multi-frequency sine-Gordon model. Nucl. Phys. B516, 675-703, 1998, hep-th/9709028

[2] M. Fabrizio, A. O. Gogolin, A. A. Nersesyan: Critical properties of the doublefrequency sine-Gordon model with applications. Nucl. Phys. B580, 647-687, 2000, cond-mat/0001227

[3] Z. Bajnok, L. Palla, G. Takács, F. Wágner: A nonperturbative study of the twofrequency sine-Gordon model. Nucl. Phys. B601, 503-538, 2000, hep-th/0008066

[4] Z. Bajnok, L. Palla, G. Takács, F. Wágner: The k-folded sine-Gordon model in finite volume. Nucl. Phys. B587, 585-618, 2000, hep-th/0004181

[5] V.P. Yurov, Al. B. Zamolodchikov: Truncated conformal space approach to scaling Lee-Yang model. Int. J. Mod. Phys. A5, 3221-3246, 1990

[6] G. Mussardo, V. Riva, G. Sotkov: Semiclassical Particle Spectrum of Double SineGordon Model, Nucl. Phys. B687, 189-219, 2004, hep-th/0402179

[7] Al. B. Zamolodchikov: Mass scale in the sine-Gordon model and its reductions. Int. J. Mod. Phys. A10:1125-1150, 1995

[8] T. R. Klassen, E. Melzer: Spectral flow between conformal field theories in $1+1$ dimensions. Nucl. Phys. B370, 511-550, 1992 